\title{
Análisis de material lítico superficial en el noreste del lago Pueyrredón-Cochrane (provincia de Santa Cruz, Argentina)
}

\author{
Damián L. BozzuTo \\ CONICET - Instituto Nacional de Antropología y Pensamiento Latinoamericano \\ dbozzuto@filo.uba.ar
}

Recibido: 31 de octubre de 2011

Aceptado: 1 de febrero de 2013

\section{RESUMEN}

La ubicación de concentraciones de material lítico en superficie puede brindar información acerca del uso de distintos sectores de un espacio determinado. El caso de estudio que se presenta aborda el análisis de material recuperado en un sector específico del noroeste de la actual provincia de Santa Cruz, Argentina. En este trabajo se discuten algunos aspectos de las distribuciones de material lítico en el noreste del lago PueyrredónCochrane sobre la base de un muestreo de material superficial. A partir del levantamiento, se evalúa el uso de las diferentes materias primas líticas y se caracterizan los espacios en los que se registraron las mayores concentraciones de artefactos. Al mismo tiempo, se evalúan posibles vías de circulación entre el sector estudiado y los valles de la vertiente occidental de la cordillera de los Andes.

Palabras clave: Patagonia, artefactos líticos, superficie, lago Pueyrredón.

\section{Surface Lithic Material Analysis at Northeast Pueyrredón-Cochrane Lake (Santa Cruz, Argentina)}

\begin{abstract}
The location of surface lithic material may provide information about uses of different areas within a certain space. The case study introduced here deals with the analysis of the material recovered in one specific area in the Northwest of what is nowadays the Santa Cruz province, in Argentina. This work analyses certain aspects of the lithic material distribution in the Northeast of the lake Pueyrredón-Cochrane based on the surface material sampling. Upon the gathering and analysis of the sampling, the use of different lithic raw materials is evaluated and the spaces where the major concentrations of artifacts were found are described. It is also assessed the possible circulation or communication between the area analyzed and the valleys of the western side of the Andes Ridge.
\end{abstract}

Key words: Patagonia, lithic artifacts, surface, Pueyrredón Lake.

Sumario: 1. Introducción. 2. Antecedentes. 3. Planteamiento del problema. 4. Objetivos y organización del trabajo. 5. Metodología. 6. Estructura de la muestra y resultados. 7. Discusión. 8. Perspectivas de futuro. 9. Referencias bibliográficas.

\section{Introducción}

El estudio que se presenta forma parte de un proyecto de investigación más amplio que está orientado a discutir el proceso de colonización, el manejo de los recursos y las interacciones de las sociedades cazadoras-recolectoras de la región noroeste de la provincia de Santa Cruz. Esta discusión se aborda por medio del estudio de diferentes tipos de evidencias procedentes del sector noreste del lago Pueyrredón-Cochrane.

Uno de los fines de este proyecto general es estudiar el flujo de información, los rangos de acción y posibles modelos de movilidad microregional entre poblaciones 
de la cuenca de los lagos Pueyrredón-Posadas-Salitroso y zonas aledañas. Al mismo tiempo se busca establecer comparaciones con otras cuencas, como por ejemplo la del lago Burmeister, en el sector sur del Parque Nacional Perito Moreno.

En este contexto, el objetivo general de la investigación que se desarrolla aquí consistió en realizar un aporte al conocimiento de la dinámica de ocupación del espacio en el sector noroeste de la provincia de Santa Cruz. Este objetivo se abordó a partir de caracterizar la ocupación de diferentes ambientes del sector noreste del lago Pueyrredón-Cochrane por medio del análisis de la estructuración de los conjuntos líticos recuperados en superficie.

\section{Antecedentes}

La cuenca de los lagos Pueyrredón-Posadas-Salitroso ha sido estudiada en el marco de distintas investigaciones que se orientaron, inicialmente, a caracterizar y relacionar el uso de dos cuencas lacustres cordilleranas (la mencionada y la de los lagos Belgrano y Burmeister, ver Figura 1) en comparación con lo observado en el alto valle del río Pinturas (Aschero 1981-82; Aschero et al. 1992).

La cuenca de los lagos Pueyrredón-Posadas-Salitroso, en el sector norte, está ubicada a $150 \mathrm{msnm}$ mientras que la de los lagos Belgrano y Burmeister -en el sector sur- se encuentra en la cota de $900 \mathrm{~m}$. Este contraste en las alturas de ambos espacios redunda en variaciones respecto de la rigurosidad del clima en un ambiente y otro. En relación con las diferencias mencionadas, se esperaba un desfasaje en los momentos de parición del guanaco (Lama guanicoe) entre ambos sectores. A su vez, a partir de este desfasaje habría resultado en una ventana temporal más amplia para la caza de los guanacos recién nacidos - cuya piel era utilizada para la confección de quillangos (capas)- en un circuito que incluyera a ambos sectores (Aschero 1981-82).

Por otra parte, en las zonas cordilleranas más cercanas al bosque habrían estado disponibles otro tipo de recursos propios de este ambiente como por ejemplo el huemul (Hippocamelus bisulcus) y una variedad de especies vegetales (Aschero 198182; Aschero et al. 1992).

En un primer momento, se abordaron trabajos en el área del Parque Nacional Perito Moreno (PNPM, Área Río Belgrano, Figura 1). En este sector se identificaron ocupaciones que abarcan desde alrededor de 10.000 años AP hasta el siglo XVIII, aunque no se encontraron materiales europeos en los sitios trabajados (Aschero et al. 2005). Con posterioridad, se iniciaron investigaciones en el sitio Cerro de los Indios 1 (CI1) que dieron un panorama general acerca de las características de las ocupaciones en el sector central de la cuenca de los lagos Pueyrredón-Posadas-Salitroso (Aschero et al. 1999). Más adelante se incluyeron otras áreas, por ejemplo los alrededores del lago Salitroso, ubicado en el sector este de la cuenca (ver Figura 2) (Goñi 2000-02; Goñi y Barrientos 2004).

A partir de los trabajos mencionados, se planteó un esquema de uso complementario de sectores altos y bajos para el Holoceno Tardío en una escala regional (Goñi 2000-02). En este modelo se postula, para los sectores bajos de la cuenca -en la zona del lago Salitroso-, un uso residencial y semi-permanente. Esto se fundamenta en la 


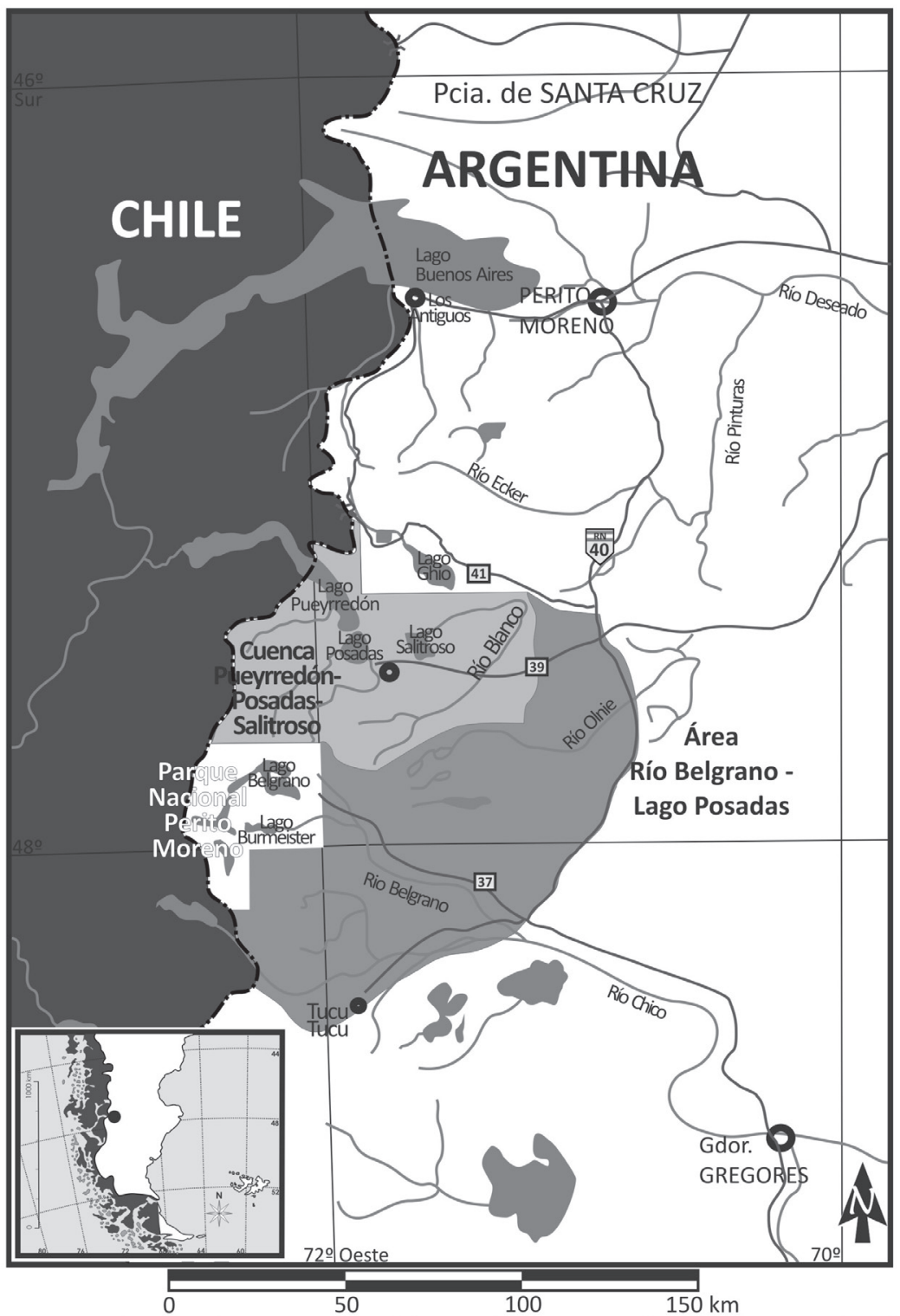

Figura 1: Ubicación del área de investigación.

estructura de recursos y las condiciones ambientales más benignas para la temporada invernal, que hacen posible una permanencia más prolongada. A su vez, se basa en que el registro arqueológico de la parte baja de la cuenca tiene una alta riqueza y una representación importante de tecnología cerámica, estructuras de entierro y artefactos de molienda (Cassiodoro 2008). Por el contrario, para los sectores altos -como Pampa del Asador, por ejemplo- las condiciones ambientales son desfavorables durante 


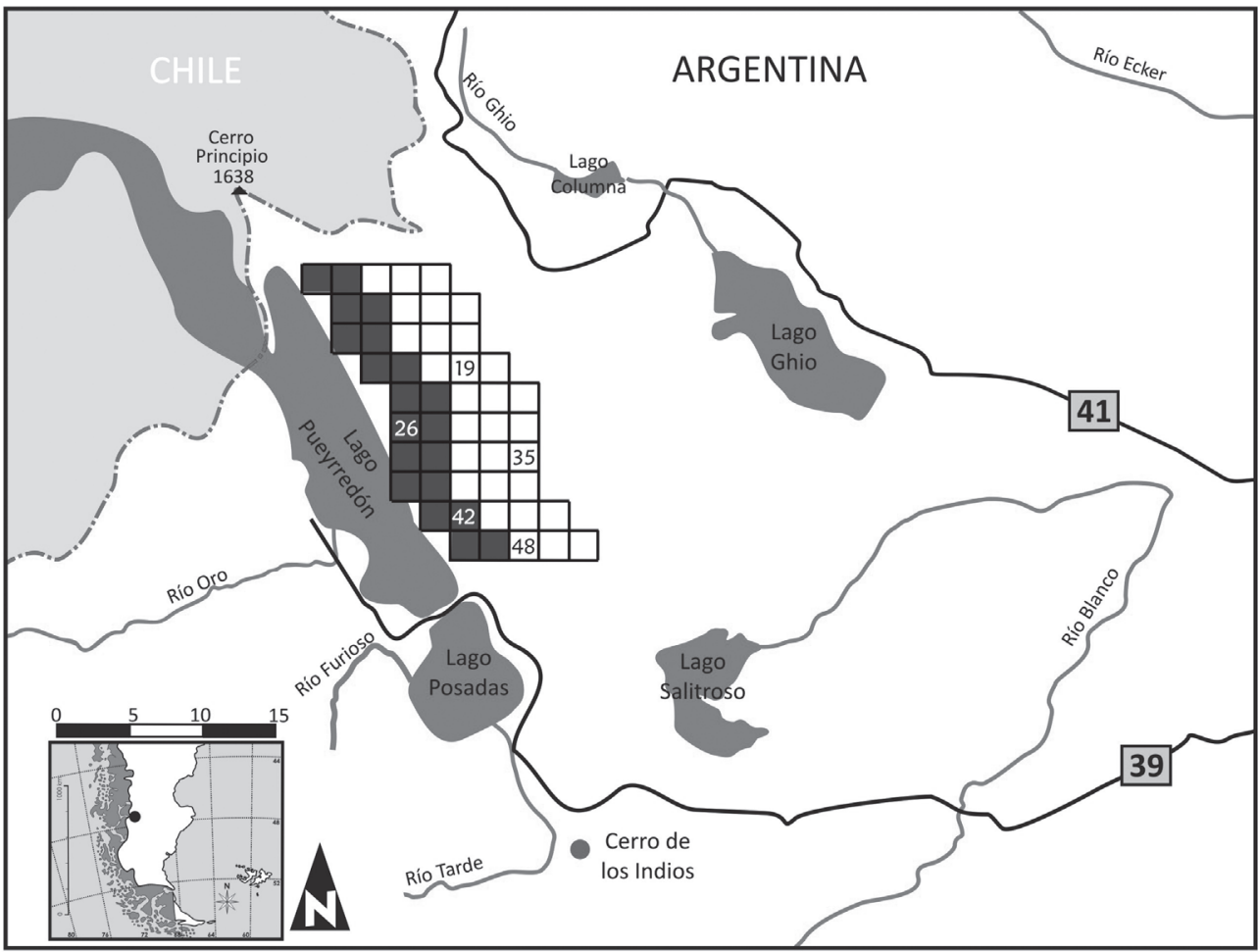

Figura 2: Ubicación del sector estudiado en la cuenca de los lagos Pueyrredón-Posadas-Salitroso.

una buena parte del año y entre las evidencias materiales registradas no se encuentran enterratorios, concentrándose los hallazgos principalmente en parapetos, que no cuentan con cerámica ni con artefactos de molienda.

En caso de darse en el sector noreste del lago Pueyrredón un contexto similar al observado en las áreas bajas de la cuenca, esta situación debería dejar como reflejo algún tipo de evidencia de ocupaciones más residenciales o semi-permanentes -en el sentido de los hallazgos mencionados para el lago Salitroso, como por ejemplo estructuras de entierro o tecnología cerámica-. Este tipo de ocupaciones debería hallarse entonces en las áreas más cercanas a la costa actual del lago. Este tipo de evidencias, hasta el momento, no ha sido encontrado.

En este contexto, a partir del año 2005 el proyecto de investigación que enmarca a este trabajo, abordó un sector específico de la sub-área Lago Posadas, ubicado en la costa del lago Pueyrredón-Cochrane (Figura 2). El objetivo inicial fue identificar y explicar los inicios y la consolidación del proceso de colonización en ámbitos perilacustres del noroeste de la provincia de Santa Cruz. El primer paso propuesto fue la generación de información para el sector noreste del lago Pueyrredón y el establecimiento de comparaciones con la información disponible para otros sectores del área de investigación, por ejemplo el PNPM, CI1 y lago Salitroso. 


\section{Planteamiento del problema}

En el marco del proyecto mencionado, el aporte del trabajo que se presenta surge a partir del cruce de distintos aspectos. En primer lugar se tuvo en cuenta que se estaba abordando el estudio de un sector de la cuenca de los lagos Pueyrredón-PosadasSalitroso que no contaba con investigaciones arqueológicas previas. De esta forma, los trabajos iniciales estuvieron orientados a reunir información que diera cuenta de las características generales de este espacio específico.

En segundo lugar, se plantea el estudio de este sector teniendo en cuenta el modelo propuesto por Goñi y colaboradores (2004). Dentro de este modelo, nos interesa principalmente la diferenciación entre las partes bajas y las altas de la cuenca de los lagos Pueyrredón-Posadas-Salitroso. El modelo plantea que, ante el progresivo desecamiento ambiental a lo largo del Holoceno, las cuencas bajas habrían conservado agua y de esta manera habrían funcionado como concentradoras de población.

El nivel de estos lagos, que está a $150 \mathrm{msnm}$ en la actualidad, habría sido muy superior en los momentos iniciales del Holoceno (Horta y Aschero 2010). A través de los estudios geomorfológicos que se realizaron, se pudo establecer una cronología aproximada de pulsos de avance de los lagos (Horta y Aschero 2010). Esta cronología se relaciona con dos importantes fluctuaciones que habían sido identificadas previamente a partir de la presencia de sedimentos lacustres en la base de dos sondeos realizados en los sitios Alero Inclinado y Cueva Milodón Norte 2. El primer nivel, identificado en Alero Inclinado, se encuentra en la cota de $190 \mathrm{~m}$. El segundo nivel, identificado a partir del sondeo en Cueva Milodón Norte 2, corresponde a la cota de 297 m. Este último nivel parece marcar un límite cronológico ya que las dataciones de los sitios ubicados por debajo del mismo no alcanzan los 4000 años AP, mientras que los sitios ubicados por encima de este nivel cuentan con ocupaciones datadas en momentos previos, como es el ejemplo de Cueva Milodón Norte 1 (Aschero et al. 2009).

\section{Objetivos y organización del trabajo}

De acuerdo con la problemática planteada, los objetivos propuestos para esta investigación estuvieron orientados, en primera instancia, a la caracterización de los conjuntos de artefactos líticos hallados en el sector estudiado. Como primer factor de esta caracterización se buscó identificar patrones en el emplazamiento de los conjuntos en diferentes geoformas. A su vez, se buscó interpretar y relacionar estos patrones con el contexto ambiental cambiante a lo largo del Holoceno. Un aspecto adicional que se busca evaluar es el de las tendencias en el aprovechamiento de las materias primas líticas en relación con sus fuentes de obtención.

Como marco de estos objetivos y teniendo en cuenta las variaciones en los niveles lacustres, se planteó que los índices de riqueza y homogeneidad de los conjuntos recuperados en cotas altas van a ser diferentes de los registrados para los conjuntos de las cotas bajas del sector estudiado. En el caso en que las variaciones de los niveles lacustres se hayan dado a lo largo del Holoceno, esto implicaría que los espacios más elevados pudieron ser ocupados durante toda la secuencia de ocupaciones del área, 
mientras que los más bajos habrían estado disponibles periódicamente o sólo hacia los momentos más recientes.

Con el fin de enmarcar la investigación en un contexto regional, se compararon los índices de riqueza y homogeneidad del norte del lago Pueyrredón con los registrados para la cuenca del lago Burmeister, en el sector sur del PNPM. Se tomó esta área porque cuenta con un levantamiento que podía ser equiparado al que se realizó para este trabajo. Asimismo, las mayores alturas y el clima riguroso registrados en el lago Burmeister -ubicado en la cota de $900 \mathrm{~m}$ - permitirían proponer un uso más esporádico de este espacio si se lo compara con el norte del lago Pueyrredón -ubicado a 150 msnm- cuyo clima habría sido más benigno.

Los objetivos planteados y los fines propuestos para este trabajo están relacionados, a su vez, con tres ejes que guiaron el desarrollo del trabajo. Aún cuando estos ejes fueron útiles para ordenar el trabajo, inevitablemente se entrecruzan en el momento del análisis. El primer eje abordó el análisis tecno-morfológico de los conjuntos en relación con las materias primas utilizadas. El segundo eje se orientó a evaluar el tamaño de los conjuntos en relación con las clases y grupos tipológicos presentes en la muestra. El último de los ejes -estrechamente relacionado con el anterior-es el análisis de la ubicación espacial de los conjuntos en relación con su tamaño y el índice de riqueza y homogeneidad que presentó cada uno de ellos.

\section{Metodología}

La metodología utilizada para alcanzar los objetivos propuestos se separó en distintas etapas. En primer lugar, se realizó el levantamiento en campo a partir de un muestreo en el que se cuadriculó la totalidad del área de estudio en cuadros de dos kilómetros de lado. A su vez, esa rejilla fue separada en dos zonas divididas -aproximadamente- por la cota de $350 \mathrm{~m}$. Esta división responde a los espacios que habrían estado disponibles a lo largo de toda la historia de ocupación del área y los más bajos, que pudieron estar alternadamente cubiertos por los avances de los cuerpos lacustres. A partir de esta cuadrícula separada en dos zonas se realizó un muestreo estratificado al azar en el que quedaron seleccionados los cuadros de muestreo que se indican con números en la Figura 2.

Cada uno de los cuadros seleccionados fue explorado por medio de cuatro transectas orientadas en sentido este-oeste. Dentro de cada transecta se recolectaron los hallazgos identificados, registrando también información acerca de cobertura vegetal, pendiente del terreno, presencia de restos óseos y el contexto de los hallazgos. Consideramos hallazgos aislados cuando se recuperaron una o dos piezas en una superficie de $25 \mathrm{~m}^{2}$ (Borrero et al. 1992), en los casos en que se presentaron tres o más hallazgos, fueron considerados concentraciones. Algunas de las concentraciones que se identificaron se ubicaban agrupadas en espacios acotados. Aún cuando estaban separadas unas de otras, se relacionaban con un único rasgo topográfico -por ejemplo una laguna- y por lo tanto se decidió reunirlas en conjuntos que se denominaron agrupaciones.

Los artefactos recuperados en cada una de las transectas fueron analizados de acuerdo con los lineamientos metodológicos propuestos por Aschero $(1975,1983)$. A 
Cuadro 1: Estructura de la muestra.

(En la categoría de desechos se incluye a los enteros fracturados con y sin talón).

\begin{tabular}{lcccccr}
\hline \multicolumn{1}{c}{ Clase Tipológica } & Cuadro 19 & Cuadro 26 & Cuadro 35 & Cuadro 42 & Cuadro 48 & Total \\
\hline Instrumentos & 10 & 13 & 57 & 53 & 4 & 137 \\
FNRC $^{*}$ & 2 & 4 & 19 & 23 & - & 48 \\
Núcleos & 2 & - & 13 & 7 & - & 22 \\
Desechos & 98 & 183 & 508 & 472 & 53 & 1314 \\
\hline Total & 112 & 200 & 597 & 555 & 57 & 1521 \\
\hline
\end{tabular}

${ }^{*}$ Filo natural con rastros complementarios

partir del análisis tecno-tipológico se pudo registrar la diversidad de los conjuntos en cuanto a las clases y los grupos tipológicos presentes como asimismo las diferentes materias primas líticas utilizadas.

Una vez que se contó con esta información, se calcularon los índices de riqueza y homogeneidad (Shannon y Weaver 1949; Zar 1974; Pielou 1977) con el objetivo de comparar los conjuntos de distintos sectores dentro del muestreo y entre éstos y los procedentes del PNPM. Estos índices expresan la diversidad de un conjunto y al mismo tiempo minimizan -a través del índice de riqueza- el grado en que el tamaño de las distintas muestras afecta las comparaciones entre ellas (Guráieb 1999).

\section{Estructura de la muestra y resultados}

En el Cuadro 1 se presenta la estructura general de la muestra, en la que están incluidos solamente los desechos enteros y los fracturados con talón. Se tomaron solamente estas dos categorías para las cuantificaciones y comparaciones entre materias primas para evitar la sobre representación de la muestra debido a posibles múltiples fracturas de un mismo desecho.

Por otra parte, en términos generales se pudo observar que la representación de materias primas en el conjunto estudiado es acorde con la que se registra en otros sectores dentro de la cuenca de los lagos Pueyrredón-Posadas-Salitroso (Cassiodoro et al. 2004). Las rocas presentes en forma mayoritaria son: la andesita, las rocas silíceas, la obsidiana, la riolita y -por último- se generó una categoría «otras» formada por distintas litologías que se presentan con muy baja frecuencia. Entre estas últimas materias primas se encuentran la dacita, la limolita, la cuarcita y la arenisca.

Con respecto al uso de las materias primas, se pudieron observar características similares en el aprovechamiento de las rocas silíceas y la obsidiana que las separan de las restantes. Las principales diferencias se relacionan con una mayor variedad de grupos tipológicos elaborados sobre estos dos tipos de roca (Cuadro 2). Algo equivalente sucede con las formas base, ya que en este caso también las rocas silíceas y la obsidiana presentan una variedad mayor de categorías en comparación con el resto de las materias primas (Cuadro 3).

Por otra parte, cuando se consideran los grupos tipológicos representados en los conjuntos, predominan ampliamente los raspadores seguidos por las raederas. De acuerdo con lo observado a partir del ángulo medido en los raspadores, este tipo de instrumentos fue descartado en un estado de uso avanzado. Esto puede sostenerse a 
Cuadro 2: Representación de grupos tipológicos por materia prima.

\begin{tabular}{lrccccc}
\hline \multicolumn{1}{c}{ Grupos Tipológicos } & Andesita & $\begin{array}{c}\text { Rocas } \\
\text { silíceas }\end{array}$ & Obsidiana & Riolita & Otras & Total \\
\hline Frag. artef. bifacial & - & 1 & 2 & - & - & 3 \\
Raspador & - & 24 & 14 & 6 & 1 & 45 \\
Raedera & 17 & 5 & 2 & 3 & - & 27 \\
Retoque en bisel oblicuo & - & 1 & 5 & - & - & 6 \\
Cuchillo & - & 2 & 1 & - & - & 3 \\
Muesca & 3 & - & 4 & - & - & 7 \\
Punta entre muescas & - & - & 1 & - & - & 1 \\
Artefacto burilante & 1 & 2 & - & 1 & - & 4 \\
Punta proyectil & - & 1 & 5 & - & - & 6 \\
Fragmento de filo & 11 & 12 & 7 & 1 & 1 & 32 \\
Bola boleadora & 1 & - & - & - & 2 & 3 \\
\hline Total & 33 & 48 & 41 & 11 & 4 & 137 \\
\hline
\end{tabular}

Cuadro 3: Forma base por materia prima en artefactos tallados.

\begin{tabular}{|c|c|c|c|c|c|c|c|c|}
\hline & & & Andesita & $\begin{array}{l}\text { Roca } \\
\text { silícea }\end{array}$ & Obsidiana & Riolita & Otras & Total \\
\hline \multirow{7}{*}{ Lasca } & \multirow{3}{*}{ Externas } & Primaria & - & - & 2 & - & - & 2 \\
\hline & & Secundaria & - & 1 & 2 & 1 & - & 4 \\
\hline & & Dorso Natural & - & 2 & 1 & 1 & - & 4 \\
\hline & \multirow{4}{*}{ Internas } & Angular & 15 & 15 & 15 & 5 & 2 & 52 \\
\hline & & Arista & 9 & 7 & 2 & 1 & - & 19 \\
\hline & & Plana & 1 & - & - & - & - & 1 \\
\hline & & No diferenciada & 4 & 9 & 3 & 2 & - & 18 \\
\hline \multirow{2}{*}{ Hoja } & & Arista Simple & - & 6 & 4 & - & - & 10 \\
\hline & & Arista Doble & 2 & 4 & 2 & 1 & - & 9 \\
\hline \multirow{2}{*}{ Otras } & \multicolumn{2}{|c|}{ Sobre Núcleo } & - & - & 1 & - & - & 1 \\
\hline & \multicolumn{2}{|c|}{ Forma Base no dif. } & 2 & 4 & 9 & - & 2 & 17 \\
\hline Total & & & 33 & 48 & 41 & 11 & 4 & 137 \\
\hline
\end{tabular}

Cuadro 4: Ángulos medidos en raspadores enteros y fracturados (divididos por materia prima).

\begin{tabular}{llcccccc}
\hline & & Mayor $\mathbf{8 0}^{\mathbf{0}}$ & $\mathbf{7 0}^{\mathbf{a}} \mathbf{a} \mathbf{7 9}^{\mathbf{0}}$ & $\mathbf{6 0}^{\mathbf{0}} \mathbf{a} \mathbf{6 9}^{\mathbf{0}}$ & $\mathbf{5 0}^{\mathbf{0}} \mathbf{a} \mathbf{5 9}^{\mathbf{0}}$ & \multicolumn{2}{c}{ Totales } \\
\hline \multirow{2}{*}{ Rocas silícea } & Enteros & 4 & 9 & 2 & - & 15 & 24 \\
& Fracturados & 7 & 2 & - & - & 9 & \\
\hline \multirow{2}{*}{ Rbsidiana } & Enteros & 5 & 2 & - & - & 7 & 14 \\
& Fracturados & 4 & 3 & - & - & 7 & \\
\hline \multirow{2}{*}{ Otras } & Enteros & 1 & - & - & - & 1 & 6 \\
& Fracturados & 4 & 1 & - & - & 5 & \\
\hline \multirow{2}{*}{ Totales } & Enteros & - & - & - & - & 0 & 1 \\
& Fracturados & - & 1 & - & - & 1 & \\
& Enteros & 10 & 11 & 2 & - & 23 & 45 \\
& Fracturados & 15 & 7 & - & - & 22 & \\
\hline & General & 25 & 18 & 2 & - & & \\
\hline
\end{tabular}


partir de los ángulos, que tienen mayormente valores cercanos o superiores a los $80^{\circ}$, cuando ya son considerados agotados (Cuadro 4). Con las raederas sucede algo similar ya que también se registra una mayoría de ángulos cercanos a los $80^{\circ}$.

En relación con el estado de las piezas en el momento de su descarte, abandono o pérdida, es interesante observar las diferencias existentes entre las distintas materias primas cuando se considera el estado de fragmentación (Cuadro 5). Es llamativa la cantidad de instrumentos fracturados de andesita, más aún si se los compara con los de rocas silíceas, una materia prima más frágil. La diferencia en el índice de fragmentación no parece relacionarse con el espesor de los artefactos (Cuadro 6). Para esta variable también es llamativa la baja frecuencia de artefactos delgados enteros en andesita si se los compara con los de rocas silíceas y obsidiana. Las tendencias observadas son similares cuando se analizan los filos naturales con rastros complementarios y los desechos de talla. Estas características, sumadas al hecho de que haya artefactos sobre obsidiana con espesores delgados que están enteros mientras que en andesita

Cuadro 5: Fragmentación de instrumentos por materia prima.

\begin{tabular}{lrrrrrrrrrrr|rrr}
\hline & \multicolumn{2}{c}{ Andesita } & \multicolumn{2}{c}{$\begin{array}{c}\text { Roca } \\
\text { silícea }\end{array}$} & \multicolumn{2}{c}{ Obsidiana } & \multicolumn{2}{c}{ Riolita } & \multicolumn{2}{c}{ Otras } & \multicolumn{2}{c}{ Totales } \\
& \multicolumn{1}{c}{ Ent } & Frc & Ent & Frc & Ent & Frc & Ent & Frc & Ent & Frc & Ent & Frc \\
\hline Raspadores & - & - & 15 & 9 & 7 & 7 & 1 & 5 & - & 1 & 23 & 22 & 45 \\
Fragmentos de filo & - & 11 & - & 12 & - & 7 & - & 1 & - & 1 & - & 32 & 32 \\
Raederas & 2 & 15 & 2 & 3 & 2 & - & 2 & 1 & - & - & 8 & 19 & 27 \\
Muesca & 1 & 2 & - & - & - & 4 & - & - & - & - & 1 & 6 & 7 \\
Punta proyectil frag. & - & - & 1 & - & 1 & 4 & - & - & - & - & 2 & 4 & 6 \\
Retoque en bisel oblicuo & - & - & - & 1 & 2 & 3 & - & - & - & - & 2 & 4 & 6 \\
Artefacto burilante & 1 & - & 2 & - & - & - & - & 1 & - & - & 3 & 1 & 4 \\
Cuchillos & - & - & 2 & - & - & 1 & - & - & - & - & 2 & 1 & 3 \\
Bola frag. & - & 1 & - & - & - & - & - & - & - & 2 & - & 3 & 3 \\
Artefacto bifacial frag. & - & - & - & 1 & - & 2 & - & - & - & - & - & 3 & 3 \\
Puntas entre muescas & - & - & - & - & 1 & - & - & - & - & - & 1 & - & 1 \\
\hline Total & 4 & 29 & 22 & 26 & 13 & 28 & 3 & 8 & - & 4 & 42 & 95 & 137 \\
\hline
\end{tabular}

Cuadro 6: Espesor y estado de intrumentos.

\begin{tabular}{llcccccc}
\hline & Estado & $\begin{array}{c}\text { Muy } \\
\text { delgado }\end{array}$ & Delgado & Grueso & $\begin{array}{c}\text { Muy } \\
\text { grueso }\end{array}$ & \multicolumn{2}{c}{ Totales } \\
\hline Andesita & Enteros & - & 3 & 1 & - & 4 & 32 \\
& Fracturados & 3 & 13 & 11 & 1 & 28 & 48 \\
\hline \multirow{2}{*}{ R. silícea } & Enteros & 3 & 13 & 6 & - & 22 & 41 \\
& Fracturados & 3 & 18 & 5 & - & 26 & \\
\hline Obsidiana & Enteros & 1 & 11 & 1 & - & 13 & 4 \\
& Fracturados & 8 & 18 & 2 & - & 28 & \\
\hline Riolita & Enteros & - & 2 & 1 & - & 3 & 11 \\
& Fracturados & 1 & 3 & 4 & - & 8 & \\
\hline Otras & Enteros & - & - & - & - & - & 2 \\
& Fracturados & - & 1 & 1 & - & 2 & \\
\hline Totales & Enteros & 4 & 29 & 9 & 0 & 42 & \\
& Fracturados & 15 & 53 & 23 & 1 & 92 & \\
& General & 19 & 82 & 32 & 1 & 134 & \\
\hline
\end{tabular}


-que tiene mayor tenacidad- los instrumentos están en su mayoría fracturados, lleva a pensar que estas fracturas, en principio, no responderían a factores post-depósito.

Los desechos presentan una situación similar a la expuesta cuando se analiza la fragmentación. En el Cuadro 7 se puede ver el estado de los desechos para cada materia prima. En este caso también es llamativa la alta fragmentación en los desechos de andesita en comparación con las restantes materias primas. En este punto es importante recordar que para el resto de los análisis sobre desechos, fueron considerados únicamente los enteros y los fracturados con talón, que suman un total de 567.

En el Cuadro 8 pueden verse los tipos de desecho por materia prima, se destaca que están presentes prácticamente las mismas categorías para todos los tipos de roca registrados. Sin embargo, se mantiene lo observado en los artefactos retocados en cuanto a una mayor diversidad para el caso de las rocas silíceas y la obsidiana. Respecto de los tamaños, se puede observar una leve tendencia que se centra hacia los tamaños mediano pequeños. La presencia de artefactos de tamaño muy grande junto con la ausencia de desechos en esta categoría sugieren la elaboración de instrumentos en espacios diferentes al explorado.

Los datos acerca de la frecuencia de los distintos grupos tipológicos brindaron la información para calcular los índices de riqueza y homogeneidad de cada cuadro. En este punto es útil hacer algunas aclaraciones sobre la información que brindan los índices utilizados. El índice de riqueza refleja la probabilidad de que un elemento cualquiera de un conjunto se ubique en una determinada categoría. Si todos los elementos

Cuadro 7: Estado de los desechos por materia prima.

\begin{tabular}{lrrrrrr}
\hline & Andesita & $\begin{array}{c}\text { Roca } \\
\text { silícea }\end{array}$ & Obsidiana & Riolita & Otras & Total \\
\hline Enteros & 71 & 101 & 86 & 35 & 20 & 313 \\
Fract. con talón & 97 & 47 & 60 & 41 & 9 & 254 \\
Fract. sin talón & 354 & 117 & 175 & 81 & 20 & 747 \\
\hline Total & 522 & 265 & 321 & 157 & 49 & 1314 \\
\hline
\end{tabular}

Cuadro 8: Tipos de lasca u hoja en desechos enteros y fracturados con talón.

\begin{tabular}{|c|c|c|c|c|c|c|c|c|}
\hline & & & Andesita & $\begin{array}{c}\text { Roca } \\
\text { silícea }\end{array}$ & Obsidiana & Riolita & Otras & Total \\
\hline \multirow{9}{*}{ Lascas } & \multirow{3}{*}{ Externas } & Primaria & 3 & 2 & 4 & 1 & - & 10 \\
\hline & & Secundaria & 4 & 3 & 8 & 5 & 2 & 22 \\
\hline & & Dorso natural & 3 & 8 & 11 & 1 & 2 & 25 \\
\hline & \multirow{6}{*}{ Internas } & Angular & 110 & 89 & 93 & 44 & 17 & 353 \\
\hline & & Arista & 33 & 31 & 23 & 21 & 5 & 113 \\
\hline & & Plana & - & - & 2 & - & 1 & 3 \\
\hline & & Flanco núcleo & - & 1 & - & - & - & 1 \\
\hline & & Reactivación & - & 2 & - & - & - & 2 \\
\hline & & No diferenciada & 9 & 1 & 1 & 2 & 2 & 15 \\
\hline \multirow{2}{*}{ Hojas } & & Arista simple & 2 & 1 & 2 & 1 & - & 6 \\
\hline & & Arista doble & 4 & 10 & 2 & 1 & - & 17 \\
\hline Total & & & 168 & 148 & 146 & 76 & 29 & 567 \\
\hline
\end{tabular}


Cuadro 9: Índices de riqueza y homogeneidad de acuerdo con los estratos.

\begin{tabular}{lccccccc}
\hline & \multicolumn{2}{c}{ Cota nivel del lago } & & \multicolumn{3}{c}{ Cota alta } & General \\
\cline { 2 - 3 } & Cuadro 26 & Cuadro 42 & & Cuadro 19 & Cuadro 35 & Cuadro 48 & \\
\hline Riqueza & 0,706 & 0,837 & & 0,724 & 0,885 & 0,452 & 0,899 \\
Homogeneidad & 0,907 & 0,775 & & 0,931 & 0,850 & 0,946 & 0,807 \\
\hline
\end{tabular}

del conjunto están en una misma categoría, el valor del índice es 0 . Por otro lado, el valor máximo del índice está en función de la cantidad de categorías presentes en el conjunto. En cuanto al índice de homogeneidad, tiene un rango de valores entre $0 \mathrm{y}$ 1. El límite inferior se da cuando la muestra tiene solamente una categoría, mientras que el límite máximo indica que los elementos están repartidos equitativamente entre las categorías presentes en el conjunto analizado.

El interés en la utilización de estos índices corresponde a la capacidad que tienen para identificar la diversidad, minimizando al mismo tiempo el efecto del tamaño de la muestra. De esta forma, se pudieron comparar los distintos conjuntos más allá de las diferentes frecuencias de hallazgos en cada uno de ellos. Los resultados obtenidos para los índices teniendo en cuenta el total de hallazgos en cada cuadro de muestreo pueden verse en el Cuadro 9. A partir de los resultados plasmados en este cuadro puede decirse que las diferencias observadas no reflejaron la división en estratos. En este sentido, se observan valores similares para los índices de cuadros que corresponden a un estrato y a otro, por ejemplo C42 y C35. Sin embargo, se pudieron observar diferencias entre los cuadros de muestreo ubicados más al norte y los localizados en la parte sur del sector estudiado.

Hasta aquí se comentaron los resultados de los índices tomados teniendo en cuenta el total de los conjuntos de cada cuadro de muestreo. A continuación se calcularon los índices considerando a los distintos conjuntos dentro de cada cuadro de muestreo por separado. A partir de los índices calculados de esta forma, se pudo observar que las agrupaciones concentran la mayor parte de la riqueza observada en el total de la muestra (Cuadro 10). Esta característica puede observarse mejor si se comparan los índices calculados para cada agrupación en comparación con el total del cuadro en el que está ubicada cada una de ellas. La diferencia se hace aún más notoria si se comparan los valores mencionados con los índices calculados para las concentraciones presentes dentro de cada cuadro. Esta situación marca una fuerte diferenciación entre el uso del espacio en los lugares en que se encuentran las agrupaciones en comparación con el resto de los conjuntos. Las agrupaciones presentan conjuntos que se destacan

Cuadro 10: Índices de riqueza y homogeneidad en agrupaciones.

\begin{tabular}{lccccc}
\hline & \multicolumn{2}{c}{ Total Cuadro } & & \multicolumn{2}{c}{ Agrupaciones } \\
\cline { 2 - 3 } \cline { 5 - 6 } & Cuadro 35 & Cuadro 42 & & Cuadro 35 & Cuadro 42 \\
\hline Riqueza & 0,885 & 0,837 & & 0,849 & 0,819 \\
Homogeneidad & 0,850 & 0,775 & & 0,849 & 0,907 \\
\hline
\end{tabular}


Cuadro 11: Índices de riqueza y homogeneidad en el Parque Nacional Perito Moreno y en el lago Pueyrredón.

\begin{tabular}{lccccc}
\hline & \multicolumn{2}{c}{ PNPM } & & \multicolumn{2}{c}{ Sector noreste del lago Pueyrredón } \\
\cline { 2 - 3 } \cline { 6 - 6 } & T 1-P4 & PV-3AB & & Agrupación 35 & Agrupación 42 \\
\hline Grupos tipológicos & 10 & 9 & & 10 & 8 \\
Riqueza & 0,777 & 0,820 & & 0,849 & 0,819 \\
Homogeneidad & 0,777 & 0,859 & & 0,849 & 0,907 \\
\hline
\end{tabular}

por las altas frecuencias, el grado de diversidad que presentan y porque parecerían funcionar como núcleos de actividades.

Un último aspecto que se tuvo en cuenta sobre la base de la información brindada por los índices de riqueza y homogeneidad fue la comparación de los resultados obtenidos para el lago Pueyrredón con los disponibles para el Parque Nacional Perito Moreno (PNPM) (Bozzuto 2005). En el Cuadro 11 se puede observar que la cantidad de grupos tipológicos presentes en los distintos conjuntos es similar. Para comparar estas dos áreas, seleccioné los conjuntos que presentaban las mayores diversidades dentro de cada caso. Con los índices de riqueza y homogeneidad sucede algo similar, aunque se distingue una leve tendencia hacia valores mayores en el área del lago Pueyrredón. Esta situación indica que las variaciones esperadas inicialmente sobre la base de las diferencias ambientales no pueden sostenerse, al menos, sobre la base de los artefactos líticos recuperados en este estudio. Sin embargo, sería necesario el desarrollo de estudios en mayor profundidad, como será discutido en el apartado siguiente.

\section{Discusión}

Si se retoman los objetivos propuestos inicialmente (caracterización de los conjuntos de artefactos líticos, identificación de patrones entre los conjuntos y su emplazamiento, evaluación de los patrones en relación con el contexto ambiental del Holoceno, establecimiento de tendencias en el aprovechamiento y la utilización de las rocas en relación con sus fuentes de aprovisionamiento), se puede sintetizar la información que brindó el tratamiento de cada uno de ellos. En primer lugar, la caracterización de los conjuntos de artefactos líticos a través del análisis tecno-morfológico permitió contar con la información necesaria para abordar el resto de los objetivos planteados originalmente. Al mismo tiempo, esta información permitió identificar conjuntos que presentaban frecuencias más altas que el resto.

El análisis de la ubicación de las agrupaciones, las concentraciones y los hallazgos aislados permitió la identificación de patrones en el emplazamiento de los distintos conjuntos. Se pudo observar que los conjuntos que presentan las mayores frecuencias de hallazgos se ubicaban en lugares que compartían algunas características topográficas. En términos generales, los hallazgos aislados fueron recuperados en lomadas o en zonas de pendiente media y suave, que no se encuentran asociados a lagunas y que no ofrecen reparo del viento. Los conjuntos identificados como concentraciones fueron recuperados en bordes de lagunas aisladas, que ofrecían además reparo del viento. Por otro lado, las agrupaciones se ubicaron en espacios que inicialmente eran 
similares a los anteriores pero que, a diferencia de ellos, presentaban lagunas encadenadas que cubren superficies mayores.

Al mismo tiempo, en una escala más amplia, la información obtenida a partir de las distintas variables y los índices que consideré, mostraron diferencias entre los cuadros relevados. Sin embargo, la evaluación de estos patrones en relación con el contexto ambiental del Holoceno, teniendo en cuenta principalmente las posibles variaciones en los niveles lacustres, reflejó que estas diferencias no respondieron a lo esperado a priori. En este sentido, los cuadros correspondientes a uno y otro estrato no mostraron diferencias que pudieran relacionarse con la división del área por la cota de $350 \mathrm{~m}$. Por el contrario, se observaron variaciones en un sentido norte-sur que no parecen estar relacionadas con la altura o la distancia a la costa del lago Pueyrredón. Además, las variaciones observadas cuando se analizaron los cuadros en un nivel general, se hicieron más notorias cuando se tuvieron en cuenta las agrupaciones y las concentraciones individualmente. A partir de esta separación, se pudo identificar que las agrupaciones, además de contar con las mayores frecuencias dentro del total del muestreo, concentran los niveles más altos de diversidad en sus conjuntos artefactuales.

Con respecto a las materias primas, se buscó la identificación de tendencias en el tratamiento de las rocas en relación con las fuentes de aprovisionamiento en los casos en que fue posible. En este sentido, las dos materias primas presentes en el área y para las que están identificadas las fuentes de aprovisionamiento son la andesita y la obsidiana. La fuente de andesita se ubica en la zona próxima a Cerro de los Indios, a unos $30 \mathrm{~km}$ lineales del sector relevado. Por otra parte, la fuente de aprovisionamiento de obsidiana está localizada en Pampa del Asador, a unos $70 \mathrm{~km}$ lineales del sector central del área relevada.

Como se mencionó antes, una característica llamativa acerca del tratamiento de la andesita es la alta fragmentación que presentan los instrumentos. De acuerdo con lo observado a partir de los espesores de las piezas, que no explicaría la fragmentación diferencial, esto podría deberse a un uso intensivo de estos artefactos o a su uso sobre materiales más densos. Por otra parte, sobre la base del análisis del tratamiento de la obsidiana se pudo observar una representación diferencial entre el sector sur y el sector norte del muestreo. En este sentido, resultó interesante la propuesta de César Méndez (2004). Este autor plantea que el sector noreste del lago Pueyrredón se habría ubicado en el recorrido de una de distintas vías alternativas de circulación de la obsidiana negra procedente de Pampa del Asador hacia los valles de la vertiente occidental de la cordillera de los Andes. Teniendo en cuenta esta propuesta, se controló el cuadro de muestreo 19 -el más cercano a la cordillera- para comparar la representación de la obsidiana en relación con el total del muestreo (Cuadro 12). Es interesante observar que en el cuadro 19 el porcentaje de obsidiana es más alto que para el resto de las materias primas. Esto se hace más llamativo si consideramos que la cantidad de hallazgos disminuye de sur a norte pero, pese a esta disminución en las frecuencias,

Cuadro 12: Comparación del porcentaje de representación de obsidiana y andesita entre el norte y el sur del muestreo analizado.

\begin{tabular}{lrrrc}
\hline & Obsidiana & Andesita & Otras & \multicolumn{1}{c}{ Total } \\
\hline Cuadro 19 & $43,10 \%$ & $20,69 \%$ & $36,21 \%$ & $100,00 \%$ \\
Promedio general & $26,23 \%$ & $29,07 \%$ & $44,70 \%$ & $100,00 \%$ \\
\hline
\end{tabular}


el porcentaje relativo de obsidiana aumenta. En principio, esta situación apoyaría la propuesta de Méndez acerca de una posible vía de circulación cuyo recorrido incluya el sector noreste del lago Pueyrredón.

\section{Perspectivas de futuro}

A partir de la realización de este trabajo, surgieron numerosas líneas para continuar con la investigación. Si bien no se pudieron contrastar las hipótesis propuestas inicialmente, este resultado fue útil para replantear el camino a seguir.

En primer lugar, el hecho de no haber podido identificar variaciones entre los estratos propuestos en el muestreo presenta un problema interesante. Es así que por un lado se hace necesario ajustar la cronología de variaciones en los niveles lacustres y, por el otro, mejorar la identificación de diferencias en las características de manufactura y mantenimiento de los artefactos a lo largo del tiempo. Sin embargo, la identificación de diferencias entre los cuadros de muestreo en un sentido norte-sur -que no había sido prevista en el diseño inicial del muestreo- merece la profundización del análisis de esta variación por medio del diseño de nuevos muestreos. El estudio de esta característica del registro servirá para mejorar el conocimiento acerca de la relación entre la cuenca de los lagos Salitroso-Posadas-Pueyrredón y los valles de la vertiente occidental de la cordillera. Esta línea de estudio se incluiría además en el contexto de la problemática regional respecto de la distribución de obsidiana negra procedente de Pampa del Asador.

En segundo lugar, las leves diferencias observadas entre los índices de riqueza y homogeneidad calculados para el lago Pueyrredón y los disponibles para el Parque Nacional Perito Moreno no son tan marcadas como para plantear un uso diferente de estos dos espacios. En este sentido, también se pudieron observar similitudes con respecto a los grupos tipológicos presentes en ambos conjuntos. Si bien, sobre la base de las similitudes mencionadas, puede plantearse que ambos espacios se habrían relacionado con el desarrollo de la caza y el procesamiento inicial de presas, es necesario evaluar este punto con mayor profundidad en el futuro. Es importante analizar también si las similitudes observadas se dan en el nivel de análisis de espacios específicos pero reflejan puntos particulares dentro de microrregiones con un uso espacial diferente, de acuerdo con lo propuesto por el proyecto marco y el modelo de Goñi (2000-02; Goñi y Barrientos 2004)

Otra línea de investigación que surge a partir del trabajo presentado se relaciona con el alto nivel de fragmentación de la andesita en el sector norte del lago Pueyrredón. Aún cuando ya se mencionaron algunas de las posibles causas de esta diferencia, es necesario un análisis específico de este problema. A partir de este tipo de estudio se podrán proponer otras razones posibles para la fragmentación diferencial de este tipo de roca en comparación con las restantes materias primas disponibles en el área.

Un trabajo pendiente es la incorporación de la información procedente de las excavaciones en los diferentes sitios estratificados que se trabajan en el área. El cruce de estos dos cuerpos de información permitirá ajustar el problema cronológico, por ejemplo, a través del diseño de los artefactos retocados y las formas de reducción de 
núcleos. De esta manera, en caso de poder identificar variaciones tecnológicas a lo largo del tiempo, éstas podrán ser el punto de inicio para la comparación con el material arqueológico recuperado en superficie.

En resumen, las líneas de investigación propuestas, al igual que otras que puedan surgir a futuro, servirán para tener una mejor comprensión de los cambios a través del tiempo y para integrar el sector estudiado con áreas vecinas. De esta forma se conseguirá una perspectiva con una escala espacial y temporal más amplia.

Agradecimientos: Los trabajos desarrollados fueron financiados por subsidios de la Agencia Nacional de Promoción Científica y Tecnológica (PICT 12262 - PICT 2488) y por una beca doctoral del Consejo Nacional de Investigaciones Científicas y Técnicas. Quiero agradecer en particular a quienes me guiaron, entre otras cosas, en el trabajo de doctorado: Teresa Civalero, Cristina Bellelli, Nora Franco y Carlos Aschero. Las tareas en campo fueron posibles gracias a la colaboración y buena predisposición de la Municipalidad de Perito Moreno, la Comisión de Fomento de Hipólito Yrigoyen, la familia Otamendi y el Sr. Mario Espona. Por último, la preparación y el desarrollo de este trabajo no hubieran sido posibles sin la ayuda, la guía, el intercambio de ideas y las experiencias compartidas con mis compañeros de equipo. Es así que tengo especial gratitud por todo lo que brindan y me enseñan día a día Carlos Aschero, Tere Civalero, Mariana Sacchi, Mariana De Nigris, Natalia Fernández, Sofia Tecce y Adriana Lacrouts.

\section{Referencias bibliográficas}

Aschero, Carlos A.

1975 «Ensayo para una clasificación morfológica de artefactos líticos aplicada a estudios tipológicos comparativos». Informe entregado al CONICET.

1981-82 «Nuevos datos sobre la Arqueología del Cerro Casa de Piedra». Relaciones de la Sociedad Argentina de Antropología 14 (2): 267-284.

1983 «Ensayo para una clasificación morfológica de artefactos líticos. Apéndice A y B». Cátedra de Ergología y Tecnología. Facultad de Filosofía y Letras, Universidad de Buenos Aires.

Aschero, Carlos A., Cristina T. Bellelli, M. Teresa Civalero de Biset, Rafael A. GoñI, A.Gabriela GuRÁIEB y Roberto MoLINARI

1992 «Cronología y tecnología en el Parque Nacional Perito Moreno (PNPM). ¿Continuidad o reemplazos?». Arqueología 2: 89-106. [Universidad de Buenos Aires].

Aschero, Carlos A., Damián Bozzuto, M. Teresa Civalero, Mariana De Nigris, Antonela Di Vruno, Vanina Dolce, Natalia Fernandez, Laura GonzÁlez y Paula Limbrunner

2009 «El registro arqueológico de la costa noreste del lago Pueyrredón-Cochrane (Santa Cruz, Argentina)», en Arqueología de la Patagonia: una mirada desde el último confin, M. Salemme, F. Santiago, M. Álvarez, E. Piana, M. Vázquez y M. E. Mansur, eds., pp. 919-926. Ushuaia: Editorial Utopías.

Aschero, Carlos A., Mariana De Nigris, María José Figuerero Torres, A. Gabriela Guráieb, Guillermo Mengoni Goñalons y Hugo Yacobaccio

1999 «Excavaciones recientes en Cerro de los Indios 1, Lago Posadas (Santa Cruz): nuevas perspectivas», en Soplando en el viento... Actas de las III Jornadas de Arqueología de la Patagonia, pp. 269-286. Buenos Aires - Neuquen: Instituto Nacional de Antropología y Pensamiento Latinoamericano - Universidad Nacional del Comahue. 
Aschero, Carlos A., Rafael A. Goñi, M. Teresa Civalero, Roberto Molinari, Silvana Espinosa, A.Gabriela GuRÁIEB y Cristina T. BELlELli

2005 «Holocenic Park: Arqueología del Parque Nacional Perito Moreno». Anales de Parques Nacionales 17: 71-119.

Borrero, Luis A., José L. Lanata y Beatriz N. Ventura

1992 «Distribuciones de hallazgos aislados en Piedra del Águila», en Análisis espacial en arqueología patagónica, Luis A. Borrero y José L. Lanata, eds., pp. 9-20. Buenos Aires: Ediciones Ayllu.

Cassiodoro, Gisela, A. Gabriela Guráieb, Anahí Re y Angélica Tívoli

2004 «Distribución de recursos líticos en sitios de superficie de la cuenca de los lagos Pueyrredón-Posadas-Salitroso», en Contra viento y marea. Arqueología de Patagonia, T. Civalero, P. Fernández y G. Guráieb, comps., pp. 57-69. Buenos Aires: Instituto Nacional de Antropología y Pensamiento Latinoamericano - Sociedad Argentina de Antropología.

GoÑI, Rafael A.

2000-02 «Fechados radiocarbónicos y registro arqueológico en la cuenca de los lagos Salitroso/Posadas (Santa Cruz)». Cuadernos del INAPL 19: 666-669.

GoÑI, Rafael A. y Gustavo BARRIENTOS

2004 «Poblamiento tardío y movilidad en la cuenca del lago Salitroso», en Contra viento y marea. Arqueología de Patagonia, T. Civalero, P. Fernández y G. Guráieb, comps., pp. 313-324. Buenos Aires: Instituto Nacional de Antropología y Pensamiento Latinoamericano - Sociedad Argentina de Antropología.

GurÁIEB, A. Gabriela

1999 «Análisis de la diversidad en los conjuntos instrumentales líticos de Cerro de los indios I (Lago Posadas, Santa Cruz)». Relaciones de la Sociedad Argentina de Antropología 24: 293-306.

Horta, Luis R. y Carlos A. Aschero

2010 «Evidencias de un paleolago Pleistoceno tardío-Holoceno temprano en el área del lago Pueyrredón, noroeste de la provincia de Santa Cruz», en Arqueología argentina en el Bicentenario de la Revolución de Mayo, Tomo V, J. Roberto Bárcena y Horacio Chiavazza, eds., pp. 1929-1934. Mendoza.

Méndez Melgar, César

2004 «Movilidad y manejo de recursos líticos de tres valles andinos de Patagonia Centro Occidental», en Contra viento y marea. Arqueología de Patagonia, T. Civalero, P. Fernández y G. Guráieb, comps., pp. 135-148. Buenos Aires: Instituto Nacional de Antropología y Pensamiento Latinoamericano - Sociedad Argentina de Antropología.

Pielou, Evelyn C.

1977 Mathematical Ecology. Nueva York: John Wiley and Sons.

Shannon, Claude E. y Warren Weaver

1949 The Mathematical Theory of Communication. Urbana: The University of Illinois Press.

ZAR, Jerrold H.

1974 Biostatistical Analysis. Upper Saddle River, NJ: Prentice Hall. 